\title{
Aerodynamic Effect of Wheel Fairings on the Wake of a For- mula One Car
}

\author{
Liam Davkin ${ }^{1, *}$ and Craig Law ${ }^{1, * *}$ \\ ${ }^{1}$ School of Mechanical, Industrial and Aeronautical Engineering \\ University of the Witwatersrand, Johannesburg, South Africa
}

\begin{abstract}
The open-wheeled configuration of Formula One cars has been known to create unfavourable aerodynamic conditions for overtaking during races. New aerodynamics regulations are set to come into effect by the 2022 season. Early concepts suggested that covering the wheels of a car are amongst the aerodynamic design changes to be implemented. This is in contrast to the traditional configuration. This study aims to evaluate the effect that these fairings have on the behaviour of the wake behind the car. This has been achieved through computational fluid dynamics (CFD) studies which surveyed the flow in the wake, that determined any changes that are added by these fairings. The fairings were found to have altered the structure of the wake, by changing the size of the regions of low-speed airflow at different positions behind the car.
\end{abstract}

\section{Introduction}

\subsection{Background}

The aerodynamic wake behind a car has been commonly attributed as a cause for the lack of overtaking in Formula One racing. This is because the aerodynamic wake is characterised by highly turbulent air flow. Any trailing car attempting to perform an overtaking manoeuvre is forced to operate in this area. The result is a loss of aerodynamic downforce experienced by the trailing car. This trailing car is prone to understeering into corners, making it difficult to successfully perform an overtake [1]. It has been found that the open-wheeled configuration of Formula One cars greatly influences the turbulent wake [2]. The high turbulence is further increased by the rotation of the wheels. Early car concepts for the 2022 Formula One season included wake-control devices (fairings) on the front and rear wheels (as seen in Figure 1). These were supposedly introduced to clean up the air flow downstream of a car. This would aid a trailing car by exposing it to less disturbed air.

\footnotetext{
*Liam.Davkin@students.wits.ac.za

**Craig.Law@wits.ac.za
} 


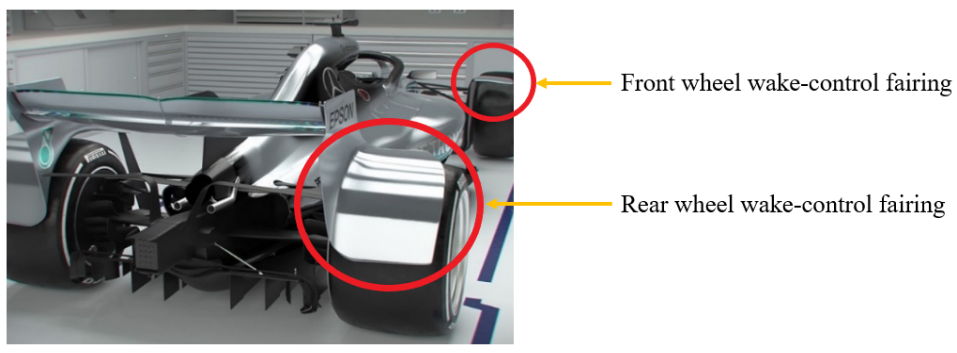

Figure 1: Concept car featuring partially covered rear wheels [3]

The car design for the 2022 Formula One season was confirmed in August of 2019, with only the front wheels making use of these wake-control fairings. There is little information in the public domain regarding the effect of wheel fairings in Formula One racing.

Previous studies have successfully characterised the turbulent wake behind a Formula One car. Watts and Watkins [4] conducted an experimental study utilising a wind tunnel scale model. Newbon et al. [5] examined the wake with the use of a numerical model. Both studies described a 'mushroom-like' wake, with strong centreline upwash and counterrotating vortices originating from each edge of the rear wing. A prominent velocity/dynamic pressure deficit was attributed to the loss of downforce by a trailing car. However, these studies only focused on open-wheeled configurations and did not implement rotating wheels. Wäschle [6] successfully demonstrated through a numerical study, the difference in wake structure for both stationary and rotating wheels. The rotating wheels reduced the size of the wake behind a car by allowing for additional energy to be transferred. These previous studies thus showed that the wake behind an open-wheeled car is highly turbulent, with regions of low flow velocity.

\subsection{Objective of Study}

This study aimed to evaluate the effect rear wheel fairings have on the wake behind a Formula One car. A survey of the velocity components in the flow field behind a Formula One car model, with various rear wheel configurations, provided data that was used to understand the effect that rear wheel fairings have on the flow field.

\section{Method}

The objective was achieved with CFD models, that surveyed the velocity components in the wake of a Formula One car, with and without wheel fairings. The method, results and conclusions pertaining to the CFD study are outlined in this paper.

\subsection{Car Model}

A generic Formula One car model was used in the CFD study (illustrated in Figure 2(a)). This model was based on an openly published computer-aided design (CAD) model [7] that was simplified in order to allow for easier scale model manufacturing. All modifications were made utilising Autodesk Inventor 2020. The model was without any unique aerodynamic features that may pertain to a car designed by a particular team. The model contained all primary downforce producing components, namely the front wing, rear wing and diffuser. 
The front and rear wings were simplified for manufacturing purposes and thus do not produce the same magnitude of downforce as would be seen in a competition vehicle. However the flow-field that results from the simplified wings should still be qualitatively similar.

Two fairing concepts were attached to the rear wheel of the car. These fairings began at the front of the wheel, at a clockwise angle of $30^{\circ}$ from the horizontal mid-plane. The fairings then extended circumferentially to a clockwise angle of $180^{\circ}$ to the back of the wheel. The dimensions for each fairing concept remained the same. A partial fairing concept had no cover over the sidewall of the wheel, whereas the full fairing concept did (shown in Figure 2(b)).

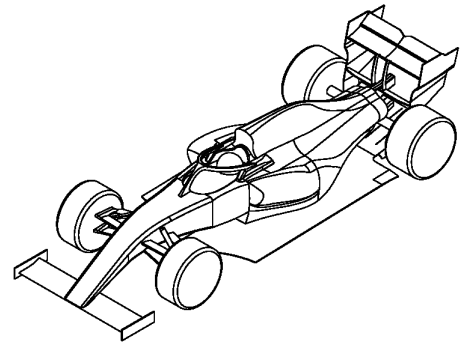

(a)
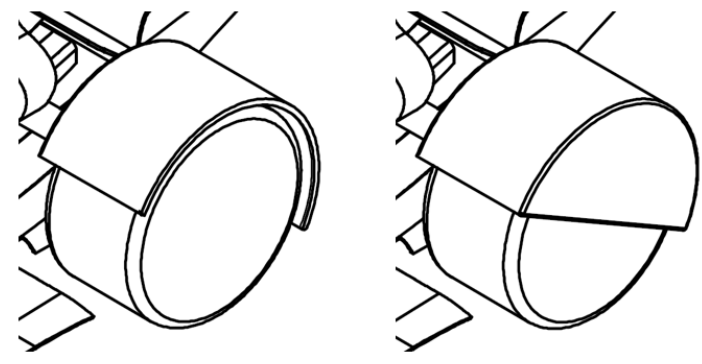

(b)

Figure 2: (a) Car model used for CFD study (without wheel fairings), (b) Partial (left) and full (right) rear wheel fairing concepts used in presented study

\subsection{Domain}

The mid-plane of the car was modelled as a symmetry plane, combined with the far fields to the top and side of the car. A velocity inlet and pressure outlet were set ahead of, and behind the car respectively. The ground plane and the surfaces of the car were modelled as smooth walls. The length of the domain was set as multiples of the length of the car $(L)$, chosen as the model characteristic length. The domain extended ahead of the car by a distance of $4 L$, and behind the car by $6 L$. The height of the domain was set at $6 h_{c}$ (equivalent to $\sim 1.28 L$ ), with $h_{c}$ being the height of the car. The width of the domain was set at $5 w_{c}$ (equivalent to $0.94 L$ ), with $w_{c}$ being the width of the vehicle. A domain with the implemented dimensions is recommended by Siemens [8] to produce a blockage ratio of less than $1 \%$, thus preventing the flow-field around the car from being affected by the domain boundary conditions.

\subsection{Mesh}

The mesh (shown in Figure 3) was composed solely of hexahedral cells, with 5 layers of prism cells at the car surfaces. A wall $y^{+}$value of 50 was chosen (i.e. the log-law region of the turbulent boundary layer) for the height of the first cell. The resolution of the boundary layer was not considered to be of primary importance in the study, as the main focus was on the wake behind the vehicle. This wall treatment was thus chosen in an attempt to minimise the cell count and hence reduce computational times. The chosen value lies within the recommended range for the selected turbulence model. Prism layer cells were disabled for the domain boundaries. Refinement zones were added immediately downstream of the car in order to accurately capture the flow properties of the wake. These zones are identified in Figure 3 . The resultant meshes were relatively coarse, consisting of $\sim 4$ million cells. 


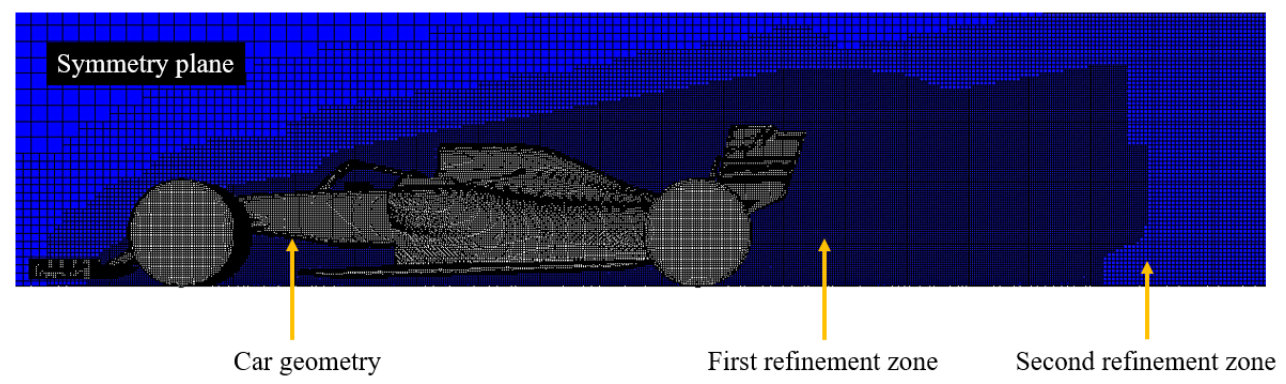

Figure 3: Side view of hexahedral mesh, showing symmetry plane and refinement zones

\subsection{CFD Model Setup}

The CFD software package used was Simcenter STAR-CCM+ 2020.3.1 (15.06.008). Only steady state simulations are presented in this study. A coupled solver was chosen in order to reach convergence faster, at the expense of more computational resources. Air density was assumed to be constant at all times. The SST $k-\omega$ turbulence model was employed as this is the recommended turbulence model for studies pertaining to external vehicle aerodynamics [9]. The value of $a_{1}$ was changed from the default of 0.31 to 1 . The realisability coefficient was set at 1.2. This setup originates from a recent study by Evans et al. [10] that showed improved stability and convergence using these values. Grid sequencing initialisation (initialisation with an inviscid solution) was activated for all simulations in order to speed up convergence. The inlet air velocity was set to $60 \mathrm{~m} / \mathrm{s}$. This was based on a rounded off average speed of $59.8 \mathrm{~m} / \mathrm{s}$ amongst all Formula One circuits in the 2019 season [11]. The outlet was set at a gauge pressure of $0 \mathrm{~Pa}$. The ground was assigned a vector speed of 60 $\mathrm{m} / \mathrm{s}$ in the same direction as the airflow. This was to prevent the formation of a boundary layer on the ground plane. Both wheels were assigned a rotational speed that corresponds to the linear speed of $60 \mathrm{~m} / \mathrm{s}$. Normalised residuals were monitored throughout the simulations, along with monitors of drag coefficient, lift coefficient, mass flow rate, and maximum velocity. The solution was considered to be converged once all normalised residuals had dropped to at least $10^{-3}$, as suggested by Celik et al. [12], and all monitored quantities had approached steady values. These criteria were satisfied for all conducted simulations, with all normalised residuals dropping below $10^{-4}$.

\section{Results and Discussion}

The results presented are representative of the three different rear wheel configurations without wheel fairings, with partial wheel fairings, and with full wheel fairings. Normalised values are presented for measurements of characteristic length, height and width of the car. Three planes perpendicular to the mid-plane of the car were considered for investigation, namely $x=1.0 L, x=1.5 L$ and $x=2.0 L$. The normalised length $x=1.0 L$ corresponds to a plane immediately aft of the car. The normalised length $x=1.5 \mathrm{~L}$ plane corresponds to a plane half a car length aft of the car. The normalised length $x=2.0 \mathrm{~L}$ plane corresponds to a plane a full car length aft of the car.

\subsection{Wake at $x=1.0 L$}

Figures 4 - 6 show that for all three fairing concepts, there existed an area of low normalised axial velocity at the rear wing of the $\operatorname{car}\left(z / h_{c} \approx 1.0\right.$, labelled as Figures $A$ in $\left.4-6\right)$. This led to 
a region of low dynamic pressure aft of the rear wing. This can be attributed to the low-speed air from the upper surface of the wing.

To the sides of the end plates, there existed a slightly increased region of normalised axial velocity. This was indicative of the formation of vortices emanating from the rear wing tips (labelled as $B$ in Figures $4-6$ ). These vortices were caused by the circulation generated by the rear wing, which created downforce. The formation of these wake vortices was further shown by the direction of the secondary flow vectors at the tips of the rear wing. These could be seen to be circulating at the rear wing tips. This circulation was in opposite directions on either side of the rear wing (i.e. the vortices were counter-rotating). The properties of these vortices were consistent with a lifting surface generating downforce. The length of the secondary flow vectors were directly proportional to the magnitude of the planar velocity. Longer vectors thus correlated with vortices with higher disturbance. These secondary flow vectors were longest for the car without any wheel fairings and shortest for the full fairing concept. The addition of wheel fairings therefore reduced the diameter of the vortices originating from the rear wing tips, by reducing the effect of the wheel disturbance. These less disturbed vortices contributed less to the upwash in the wake of a trailing car, and created favourable downforce generating conditions.

Circulation was again seen to the outside of the rear wheels $\left(y / w_{c} \approx \pm 0.7\right.$, labelled as $C$ in Figures 4 - 6) for all three concepts. These vortices originated from the rear wheels during rotation. Air close to the ground was forced outwards as the wheel rotates. The centres of these vortices contained a dynamic pressure deficit as a result of the low axial velocity of the airflow. The size of the low speed core (normalised axial velocities between 0.4 and 0.6 ) was seen to increase with the addition of both faring concepts. However, the planar velocity magnitude of these vortices were highest for the car without any wheel fairings attached. This was due to stronger inwash that was present for this configuration. These areas of airflow on the outside of the wheel are of primary concern for any trailing car. A trailing car would have to move through these wheel vortices as it attempts to pass the leading car on the side. The upwash induced by the wheel vortices reduces the effective angle of attack of the trailing car's front wing, thus reducing downforce. The front wing of the trailing car would be at such a height that it moves directly through these vortices. If a dynamic pressure deficit existed in this region, the front wing of the trailing car would lose downforce, and thus understeer into corners.

A low velocity region (normalised axial velocity $\approx 0.0$ ) existed at the exit of the diffuser of the car for all three cases (labelled as $D$ in Figures 4 - 6). This was indicative of airflow from underneath the car that expanded through the diffuser. This low velocity region was smallest for the partial wheel fairing concept, and largest for the car without any wheel fairings. The addition of wheel fairings would increase the ground effect experienced by a car by reducing the size of this low velocity region. A low velocity region at this height would result in a higher pressure on the underside of a trailing car (the space between the car and the ground), thus reducing the amount of downforce generated, while also reducing the amount of drag force experienced.

The most obvious difference between the three cases was the area immediately aft of the rear wheel $\left(y / w_{c}\right.$ between \pm 0.3 and \pm 0.5 , labelled as $E$ in Figures $\left.4-6\right)$. For the car with no wheel fairings, an area of low normalised velocity $(\approx 0.0)$ existed between $z / h_{c} \approx 0.0$ and 0.5 (i.e. half the car height). With the addition of wheel fairings, this low normalised velocity region was reduced by approximately $50 \%$. This region also moved slightly inwards. These phenomena were again observed for the full fairing configuration. Before wheel fairings were applied to the car, there existed an airflow separation point towards the top of the rear wheel. This separated flow caused a region of low dynamic pressure aft of the wheel. By adding fairings, any separated airflow was forced to flow along the path created by the fairing. 
Airflow thus exited this enclosed compartment at the bottom of the fairing. Here the air experienced possible rapid expansion, which led to a loss of dynamic pressure and thus low velocity. The addition of the wheel fairings also created a stronger downwash aft of the wheel. The addition of wheel fairings also resulted in inward secondary flow vectors close to the ground, aft of the wheel. A strong centreline upwash was present for all three cases. For all three cases, the height of the wake remained at a normalised height of approximately $z / h_{c}$ $\approx 1.0$.

Thus, the addition of fairings has changed the size and positions of the low-speed regions of airflow immediately aft of the car $(x=1.0 L)$. By reducing the size of this region towards the centreline of the car, favourable downforce generating conditions are created for a trailing car immediately behind. However, as a trailing car moves to the side of a leading car during the overtaking manoeuvre, it will encounter larger regions of low-speed airflow when fairings are applied. This creates unfavourable conditions for downforce generation as a trailing car moves closer, and thus diminishes the chance of successful overtaking in corners.

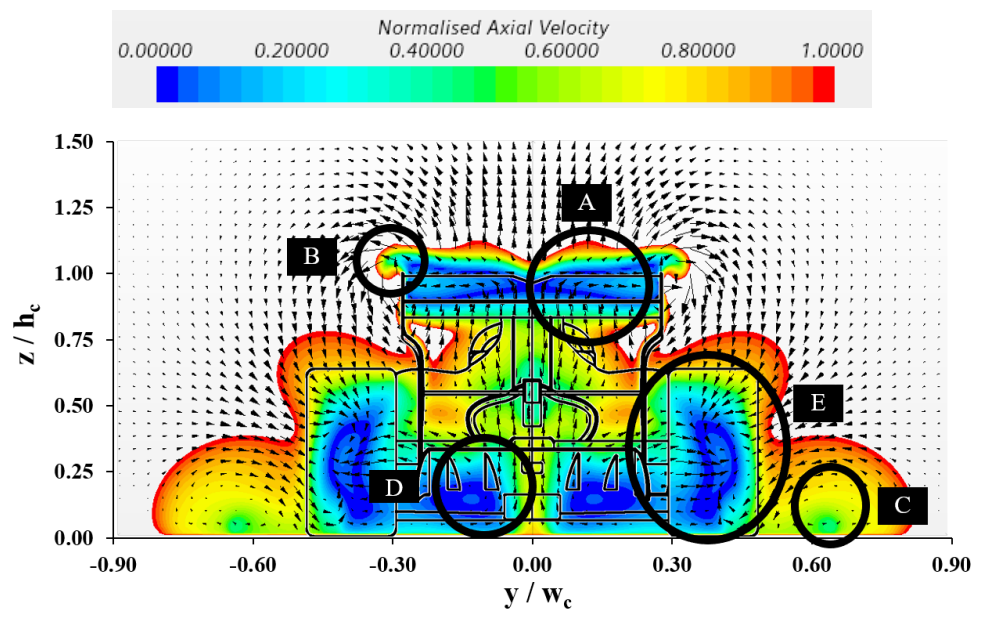

Figure 4: Normalised axial velocity contours and secondary flow vectors for no wheel fairings at $x=1.0 L$

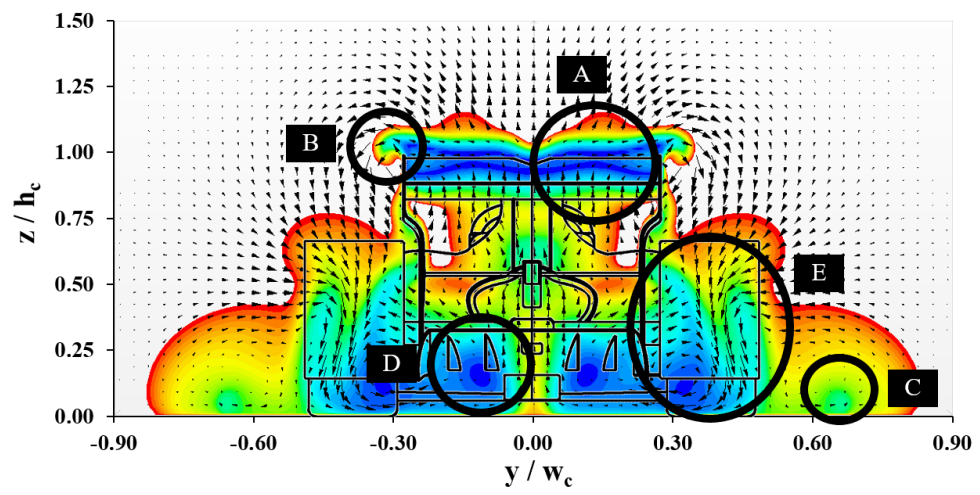

Figure 5: Normalised axial velocity contours and secondary flow vectors for partial wheel fairings at $x=1.0 L$ 


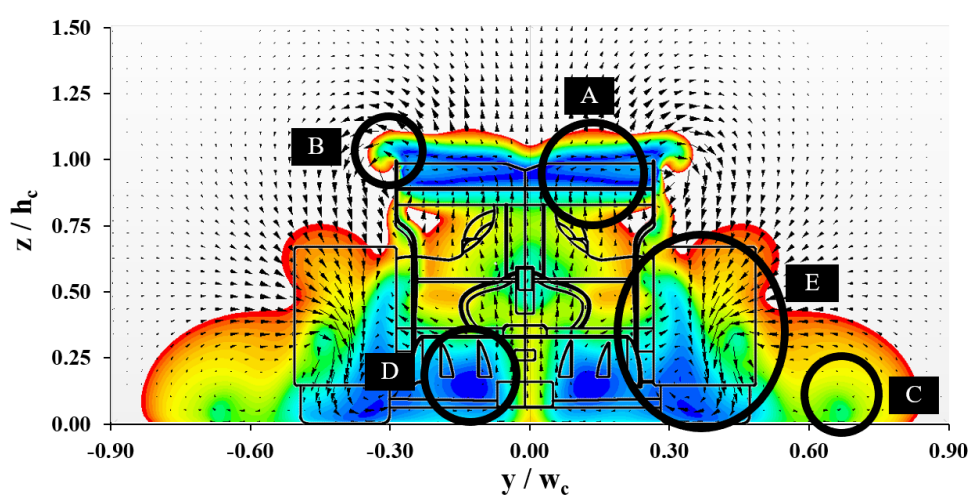

Figure 6: Normalised axial velocity contours and secondary flow vectors for full wheel fairings at $x=1.0 \mathrm{~L}$

\subsection{Wake at $x=1.5 L$}

Figures 7 - 9 show that the height of the wake increased for all three cases (labelled as $A$ ), and that the wing tip vortices had increased in size. These counter-rotating vortices (labelled as $B$ ) had formed the previously observed 'mushroom-like' wake.

Flow vectors representing the centreline upwash were influenced by the wing tip vortices and were directed diagonally upwards away from the mid-plane of the car (labelled as $C$ in Figures 7 -9). The secondary flow vectors on the outer edge of the wake tended to be directed diagonally downwards to the mid-plane of the car (labelled as $D$ in Figures 7 - 9).

Regions of low dynamic pressure were still present to the sides of the rear wheels $\left(y / w_{c} \approx\right.$ \pm 0.6 , labelled as $E$ in Figures 7 - 9). These regions moved slightly inwards to the mid-plane of the car. The car without any wheel fairings produced this smallest region. The addition of wheel fairings extended the thickness of this low pressure region to the region aft of the rear wheels. The addition of partial wheel fairings increased the thickness of this region by approximately $67.5 \%$. This increase remained approximately constant between the two fairing concepts. The presence of wheel fairings also decreased the normalised axial velocity in this region.

However, along the centreline, there existed regions of low velocity airflow for all three cases (labelled as $F$ in Figures 7 - 9). The position of this region was different between the three cases. For a car with no wheel fairings, the centre of this low velocity region existed at a normalised height of $z / h_{c} \approx 0.45$. With the addition of partial wheel fairings, the centre height was increased to a normalised height of $z / h_{c} \approx 0.575$ (an increase in height of $27.8 \%$ ). With the addition of full wheel fairings, the centre height was increased to a normalised height of $z / h_{c} \approx 0.75$ (an increase in height of 66.7\%). An increase in height may have adverse conditions for downforce generation by a trailing car. Oncoming airflow to the rear wing of the trailing car would be at a lower velocity when a leading car has full wheel fairings.

The region around the centreline of the car formed a low velocity column for the entire height of the car (labelled as $G$ in Figures 7 - 9). This column was thickest for a car without wheel fairings, with a normalised thickness of approximately 0.9. This column thickness was particularly prominent at a height corresponding to the upper half of the rear wheel. With partial and full wheel fairings, this normalised thickness was reduced to approximately 0.6. This represented an approximate thickness decrease of approximately $30 \%$. The fairings prevented interaction between the airflow around the rear wheel and the airflow around the 
rest of the car. This interaction was thus delayed and occurred further aft of the car, resulting in low velocity regions.

Therefore, at the distance of $\mathrm{x}=1.5 \mathrm{~L}$, the wake began to develop the previously seen 'mushroom-like' wake. Columns of low-speed air existed along the centreline of the car, with the region of lowest velocity having increased with the addition of wheel fairings. The region of low velocity airflow moved inwards, which would reduce the dynamic pressure deficit of a trailing car attempting an overtaking manoeuvre, thus creating favourable downforce conditions.
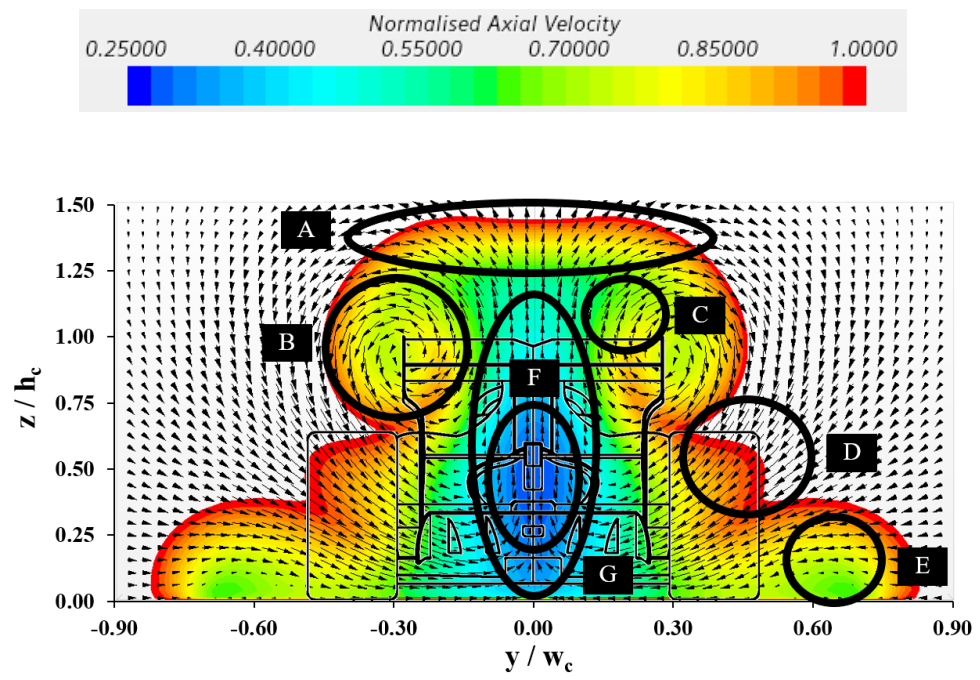

Figure 7: Normalised axial velocity contours and secondary flow vectors for no wheel fairings at $x=1.5 \mathrm{~L}$

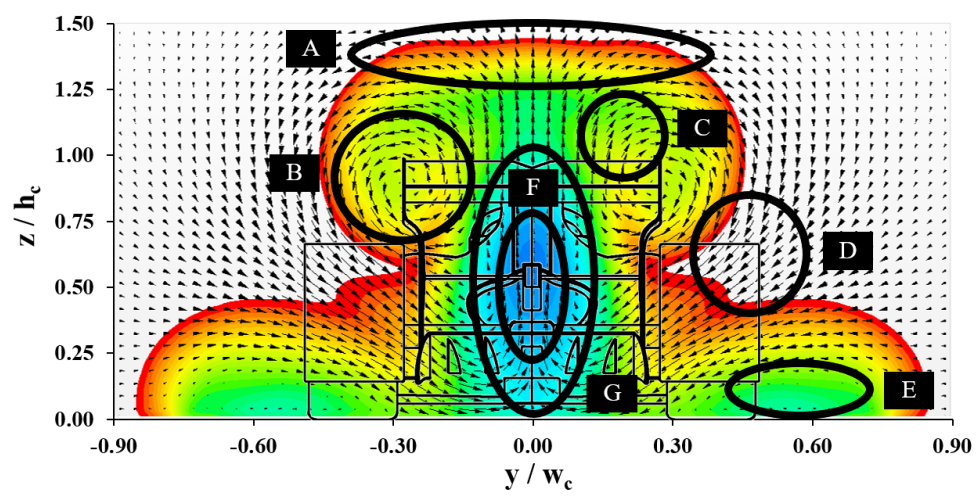

Figure 8: Normalised axial velocity contours and secondary flow vectors for partial wheel fairings at $x=1.5 \mathrm{~L}$ 


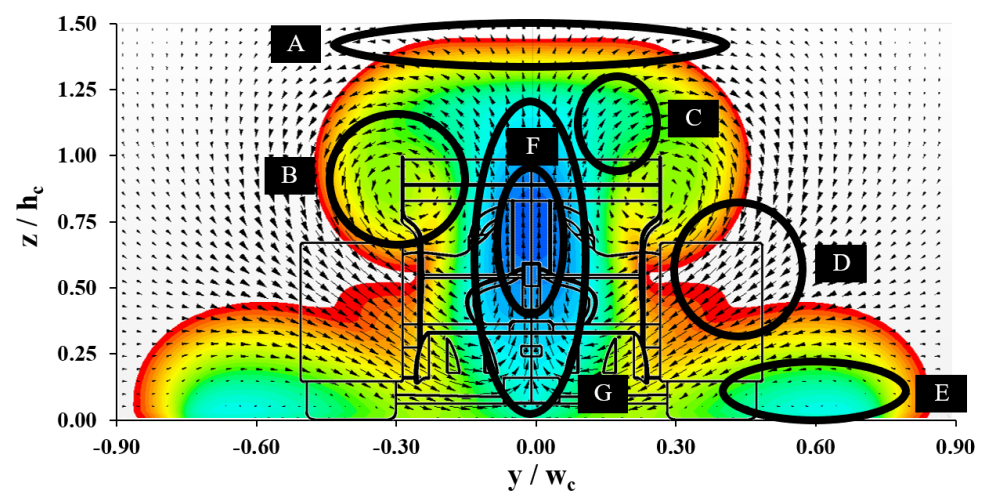

Figure 9: Normalised axial velocity contours and secondary flow vectors for full wheel fairings at $x=1.5 \mathrm{~L}$

\subsection{Wake at $x=2.0 L$}

Figures 10 - 12 show that the normalised height of the 'mushroom-like' wake increased beyond $z / h_{c} \approx 1.50$ (labelled as $A$ ).

Regions of low normalised axial velocity still existed to the sides of the wheels for all three cases (labelled as $B$ in Figures 10 - 12). The velocities in this region were lowest for the car with wheel fairings attached.

The main difference between the three cases was the difference in structure of the lowvelocity column at the centreline of the car (labelled as $C$ in Figures $10-12$ ). For all three cases, the height of the lowest velocity region $(\approx 0.45)$ extended to a normalised height of $z / h_{c} \approx 1.25$. However, this region extended to the ground plane when no wheel fairings were attached. This resulted in a low velocity region for the entire height of the car. The addition of wheel fairings caused this column of low velocity air to increase in magnitude. For the partial wheel fairing configuration, this region correlated to a normalised axial velocity of approximately 0.615 (an increase of $37 \%$ ). For the full wheel fairing configuration, this region correlated to a normalised axial velocity of approximately 0.725 (an increase of $61 \%$ ). If a trailing car was positioned at this distance aft of the leading car, this low velocity region would have considerable effects on downforce production. The lower the air velocity in this region, the less the downforce generated by the trailing car would be. The addition of wheel fairings moved this region to above the height of the car. The airflow above the height of the car would have less of an impact on the downforce production as it would not come into contact with any downforce generating components of the car.

The 'mushroom-like' wake has therefore continued to grow at a distance of $x=2.0 \mathrm{~L}$. The addition of wheel fairings reduced the size of the column of low-speed airflow, which would create favourable downforce generating conditions immediately aft of the car. However, the regions to the side of the wheels still had a decrease in normalised axial velocity that would continue to hinder overtaking opportunities.

The results at these three planes indicate that a trailing car would be able to generate more downforce when moving immediately behind the car (with the addition of wheel fairings resulting in higher downforce production). However, as the trailing car moves to the side of the leading car, downforce production is reduced by the presence of low-speed axial velocity. As the trailing car gets closer to the car ahead, the axial velocity decreases, and downforce generation is diminished, resulting in understeer and an unsuccessful overtaking manoeuvre. 


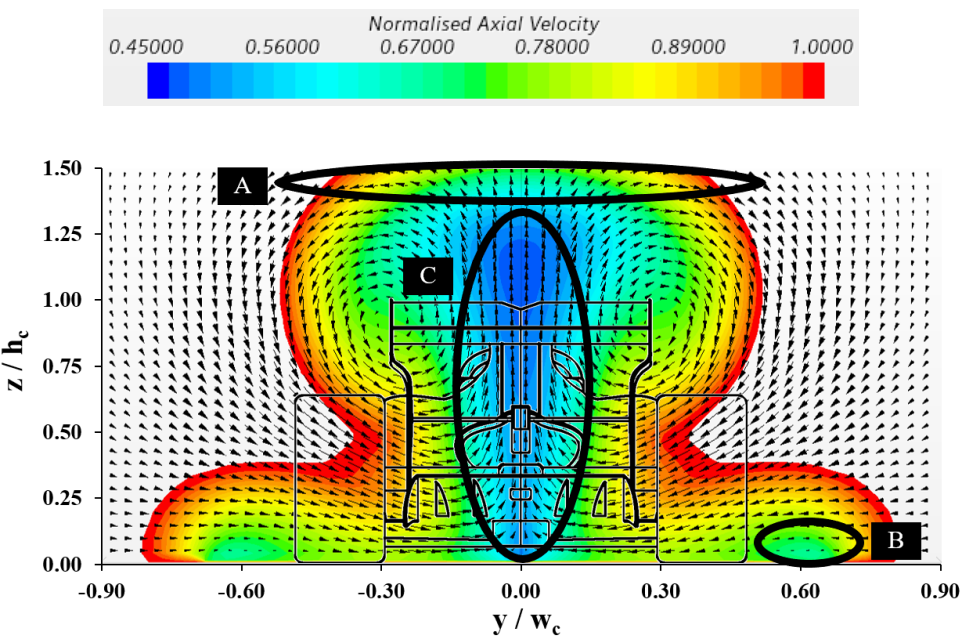

Figure 10: Normalised axial velocity contours and secondary flow vectors for no wheel fairings at $x=2.0 L$

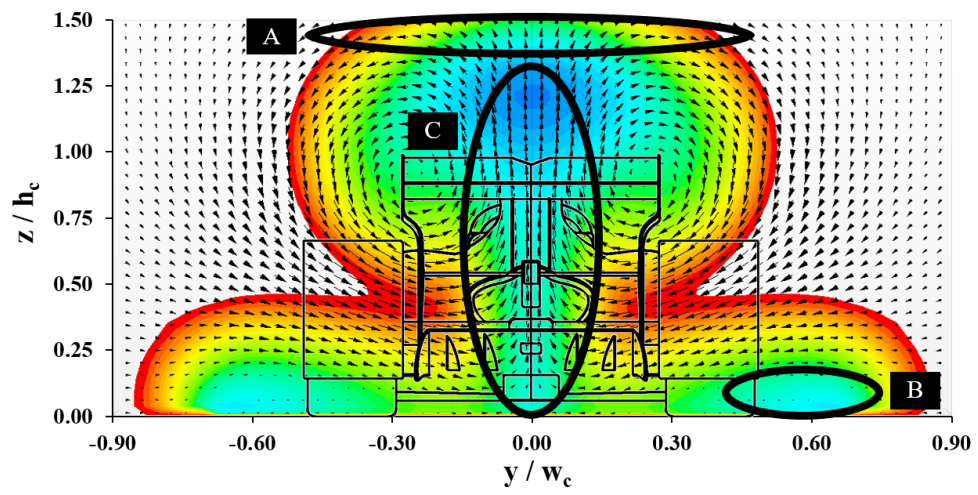

Figure 11: Normalised axial velocity contours and secondary flow vectors for partial wheel fairings at $x=2.0 L$

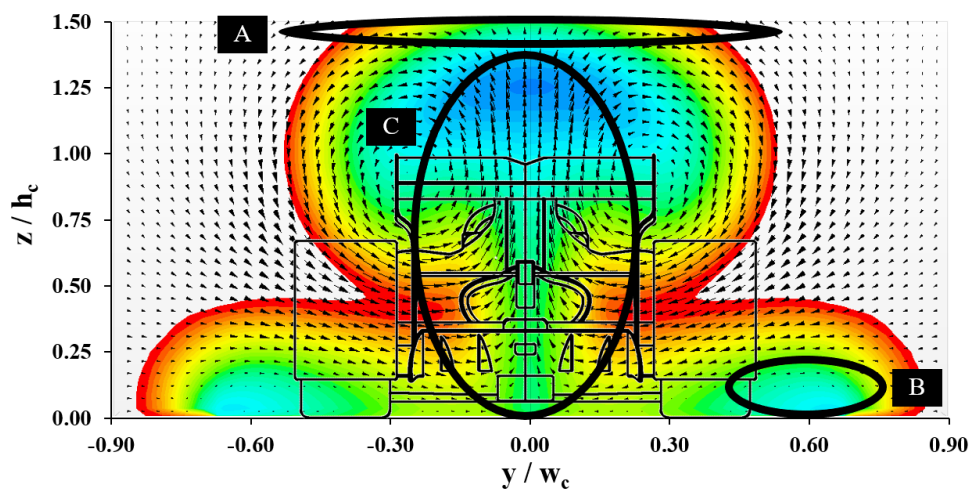

Figure 12: Normalised axial velocity contours and secondary flow vectors for full wheel fairings at $x=2.0 \mathrm{~L}$ 


\subsection{Streamlines at rear wheel}

The following streamlines are coloured by velocity magnitude and are seeded from the rear wheel. Figure 13(a) shows that as air flowed around the wheel, it did not remain attached, and separated at some point downstream of the vertical mid-plane (labelled as $A$ ). This was the expected behaviour and is analogous with the airflow around a cylinder. Aft of the separation point, there existed a large wake of low speed airflow and backflow (labelled as $B$ ). The airflow in this region was highly turbulent and chaotic as indicated by the random pattern of the streamline paths. This would create unfavourable conditions for a trailing car to generate downforce. Figure 13(b) shows that by covering the rear wheels with partial fairings, the wake region of highly chaotic airflow had been reduced (labelled as $C$ ) as the airflow was forced to follow the enclosed path created by the fairing. The airflow escaped from the sidewall of the fairing (labelled as $D$ ). This air was at higher velocity than the wake created when no wheel fairings were attached. Figure 13(c) shows that the airflow exited from the bottom of the wheel fairing (labelled as $E$ ). This resulted in rapid expansion of the air. The result was that the airflow slowed down and created a low velocity wake aft of the wheel.

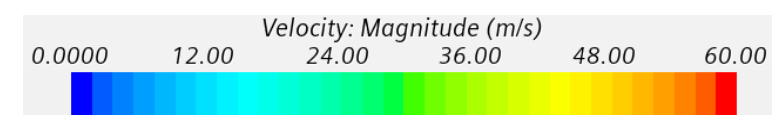

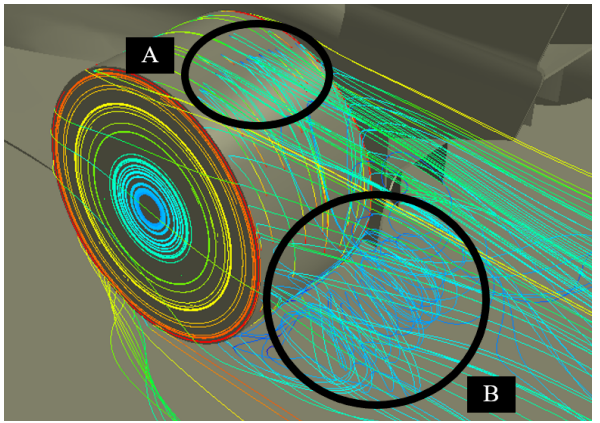

(a)

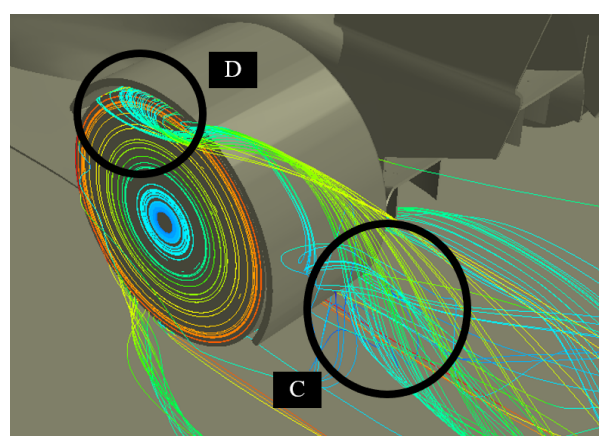

(b)

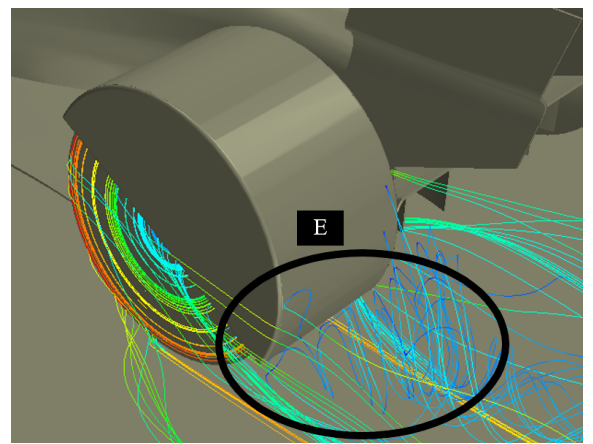

(c)

Figure 13: Streamlines for (a) no wheel fairings, (b) partial fairings, and (c) full fairings coloured by velocity magnitude 


\section{Conclusion}

This study has shown that the addition of wheel fairings had noticeable effects on the wake behind a Formula One car. The addition of wheel fairings reduced the size of the low speed airflow immediately aft of the rear wheels. The wheel fairings also concentrated the counterrotating vortices originating from the tips of the rear wing. The addition of wheel fairings increased the size of the low speed airflow to the sides of the vehicle and created unfavourable conditions for downforce production here. However, the fairings reduced the low axial velocity region at the centreline of the leading car, and also reduced the upwash, which created favourable downforce generating conditions for a trailing car. The fairings had an adverse effect on overtaking opportunities due to the need for a trailing car to always pass through the low-speed airflow (worsened by the addition of wheel fairings) to the side of the wheels of the leading car whilst overtaking. This suggested a possible explanation as to why the confirmed 2022 design only makes use of partial fairings on the front wheels of the car. Higher resolution studies are being conducted, along with wind tunnel measurements in order to provide validation data. Further work also is being undertaken that focuses on the aerodynamic performance of a trailing vehicle.

\section{References}

[1] C. Scarborough. F1: Why No Overtaking and Are Bigger Wings the Answer? (2017). URL: https://bit.ly/3EAEsT1 (Last accessed on 4 April 2019).

[2] J. Noble. The key questions raised by Formula 1's 2021 car concepts. (2018). URL: https://bit.ly/3CvcQga (Last accessed on 4 April 2019).

[3] A. Cooper, V. Khorounzhiy. 2021 Formula 1 concept claimed to produce five-times less dirty air. (2019). URL: https://bit.ly/391Ai3e (Last accessed on 4 April 2020).

[4] M. Watts, S. Watkins. "Aerodynamic Structure and Development of Formula 1 Racing Car Wakes", SAE Int. J. Passeng. Cars - Mech. Syst. 7(3):(2014), doi:10.4271/2014-010600.

[5] J. Newbon, D. Sims-Williams, R. Dominy. "Aerodynamic Analysis of Grand Prix Cars Operating in Wake Flows," SAE Int. J. Passeng. Cars - Mech. Syst. 10(1): (2017), doi:10.4271/2017-01-1546.

[6] A. Wäschle. "The Influence of Rotating Wheels on Vehicle Aerodynamics - Numerical and Experimental Investigations". In: SAE Technical Paper Series. (2010). doi:10.4271/200701-0107.

[7] R. Rodrigues. Ferrari SF71H. (2018). URL: https://bit.ly/2ZhdUX3 (Last accessed on 6 May 2019).

[8] Siemens. Best Practices for Vehicle Aerodynamics. (2021). URL: https://sie.ag/3CrHszs (Last accessed on 19 September 2021).

[9] Simcenter STAR-CCM+ User Guide. Siemens. (2020).

[10] S. Evans, S. Lardeau, C. Pettinelli. 'Validation of a turbulence methodology using the SST $k-\omega$ model for adjoint calculation'. In: 54th AIAA Aerospace Sciences Meeting. (2016). isbn: 9781624103933. doi: 10.2514/6.2016-0585.

[11] H. Cobb. The fastest F1 tracks on the calendar. (2020). URL: https://bit.ly/3lHA5gf (Last accessed 18 May 2020)

[12] I.B. Celik, U. Ghia, P.J. Roache, C. J. Freitas, H. Coleman, P.E. Raad. (2008). "Procedure for estimation and reporting of uncertainty due to discretization in CFD applications". Journal of Fluids Engineering, Transactions of the ASME, 130(7), 0780011-0780014. https://doi.org/10.1115/1.2960953 